\title{
Validação de um modelo viscoelástico para análise de estruturas de concreto submetidas a cargas de longa duração
}

Validation of a viscoelastic model for analysis of concrete structures submitted to long-term loads

\author{
L. C. de L. Pinheiro ${ }^{1 *}$; M. de V. Real ${ }^{2}$; F. C. Magalhães ${ }^{3} ;$ M. Scherer ${ }^{4}$ \\ ${ }^{1}$ Programa de Pós-Graduação em Modelagem Computacional (PPGMC), Universidade Federal do Rio Grande \\ (FURG), 96203-900, Rio Grande-RS, Brasil \\ ${ }^{2}$ Escola de Engenharia (EE), Universidade Federal do Rio Grande (FURG), 96203-900, Rio Grande-RS, Brasil \\ ${ }^{3}$ Laboratório de Estruturas e Materiais de Construção Civil (LEMCC), Instituto Federal do Rio Grande do Sul (IFRS) \\ - Campus Rio Grande, 96201-460, Rio Grande-RS, Brasil
}

${ }^{4}$ Programa de Pós-Graduação em Engenharia Civil (PPGEC), Universidade Federal do Rio Grande do Sul (UFRGS), 90035-190, Porto Alegre-RS, Brasil

*leandroclpinheiro@gmail.com

(Recebido em 12 de janeiro de 2020; aceito em 06 de julho de 2020)

\begin{abstract}
O concreto de Cimento Portland, por ser o material de construção mais utilizado no mundo, tem o comportamento, das suas mais diversas propriedades, estudado de forma cada vez mais aprofundada. Muitos estudos sobre estruturas de concreto têm sido realizados ao longo das últimas décadas, porém, a consideração dos efeitos decorrentes da aplicação de cargas de longa duração ainda carece de melhor previsão. Os efeitos diferidos são caracterizados por deformações contínuas no elemento de concreto ao longo do tempo, podendo ocorrer devido à retração e à fluência, fenômenos que aumentam a dificuldade de representação do comportamento do material. Neste trabalho é apresentada a validação de um modelo viscoelástico para o concreto, que leva em consideração os efeitos gerados por cargas de longa duração. Os efeitos diferidos foram considerados com base no que foi apresentado no CEB-FIB Model Code 90 adaptado à teoria da solidificação. A validação do modelo é feita através de comparações com resultados experimentais. A reprodução dos experimentos é feita através do Método dos Elementos Finitos utilizando-se o software ANSYS, sendo o modelo representativo do concreto inserido através da rotina USERMAT. Os resultados mostram uma boa capacidade de representação dos resultados experimentais por parte do modelo proposto. Palavras-chave: concreto, efeitos diferidos, modelo viscoelástico.
\end{abstract}

The Portland Cement concrete, as the most used construction material in the world, has the behavior of its various properties increasingly studied. Many studies about concrete structures have been conducted over the last decades; however, the consideration of the long-term load effects still needs better knowledge. The longterm effects are characterized by continuous deformations in the concrete element over time. It may occur due to shrinkage and creep phenomena that make more difficulty representing the material behavior. This work presents the validation of a viscoelastic model for concrete, which takes into account the effects generated by long-term load. The long-term effects were considered based on what was presented in CEBFIB Model Code 90 adapted to the solidification theory. The model validation is made through comparisons with experimental results. The experiments are reproduced using the finite element method, through the ANSYS software, and the representative model of the concrete inserted through the USERMAT routine. The results show a good agreement between the proposed model and the experimental results.

Keywords: concrete, long-term effects, viscoelastic model.

\section{INTRODUÇÃO}

O concreto de Cimento Portland vem sendo, ao longo das últimas décadas, o material de construção mais utilizado no mundo impulsionado, sobretudo, por sua elevada resistência, facilidade de adequação aos mais variados formatos e pelo custo relativamente reduzido quando comparado aos demais materiais construtivos [1]. A relevância deste material aumenta quando se leva em conta sua difundida utilização em sistemas estruturais, impactando de forma direta na 
segurança das estruturas e dos usuários. Todos estes fatores fazem com que se estude, de forma cada vez mais aprofundada, o comportamento das mais diversas propriedades do concreto.

A variabilidade das propriedades do concreto, tais como valores de resistências à tração e compressão, relação tensão-deformação, retração e fluência fez com que, por muito tempo, fossem adotadas metodologias de cálculo estrutural de modo a projetar estruturas de concreto armado superdimensionadas. Nas últimas décadas houve uma evolução considerável nas metodologias adotadas, porém, ainda é constante a busca pelo aperfeiçoamento dos métodos existentes e pela proposição de métodos ainda mais refinados.

Embora os métodos empíricos tradicionais permaneçam adequados para o projeto de elementos de concreto armado, a ampla utilização de computadores e o desenvolvimento do Método dos Elementos Finitos forneceram os meios para análises de sistemas muito mais complexos de uma forma muito mais realista. Porém, o principal obstáculo para a análise através do Método dos Elementos Finitos de estruturas de concreto armado é a dificuldade na caracterização das propriedades do material [2]. Esta afirmação continua válida nos dias atuais, uma vez que fatores que antes não eram considerados passam a ter relevância no cenário de estruturas cada vez mais esbeltas.

Muitos estudos sobre estruturas de concreto têm sido realizados ao longo das últimas décadas, porém, a consideração dos efeitos decorrentes da aplicação de cargas de longa duração ainda carece de melhor previsão. Os efeitos diferidos são caracterizados por deformações contínuas no elemento de concreto ao longo do tempo, podendo ocorrer devido à retração, que independe da tensão que o elemento está submetido, e à fluência, que ocorre em decorrência de uma tensão constante aplicada. Estes fenômenos aumentam a dificuldade de representação do comportamento do material. Recentemente outros estudos $[3,4,5,6]$ têm abordado os efeitos diferidos do concreto, porém, mantendo o foco na proposição e análise de parâmetros para a determinação do coeficiente de fluência e não na validação de um método proposto em comparação com estruturas reais. Assim sendo, este trabalho tem por objetivo a validação de um modelo para análise de estruturas de concreto levando-se em consideração os efeitos gerados por cargas de longa duração.

Neste estudo adotou-se o Método dos Elementos Finitos para a análise das estruturas, através do software ANSYS. A rotina USERMAT foi utilizada para a inserção de um modelo constitutivo para o concreto considerando os efeitos reológicos, baseado no CEB-FIP Model Code 90 [7], uma vez que o modelo constante no software não representa de maneira satisfatória o comportamento deste material.

Segundo Dias (2013) [8], as soluções numéricas não lineares geralmente estão formuladas em termos de taxa. Portanto é necessário converter a lei de fluência (do tipo integral) em uma lei em termos de taxa. Isso é possível, não de forma exata, porém com uma boa aproximação, através de um modelo reológico composto por molas e amortecedores combinados. Dentre os modelos existentes, a teoria da solidificação proposta por Bazant e Prasannan (1989) [9] para modelagem da fluência e retração com auxílio de uma cadeia de Kelvin para descrever o comportamento viscoelástico do concreto, vem sendo utilizada em muitos estudos $[8,10,11,12]$ e foi adotada neste trabalho.

\section{MATERIAL E MÉTODOS}

\subsection{MODELO VISCOELÁSTICO PARA O CONCRETO}

Os efeitos diferidos no concreto podem ser explicados devido aos fenômenos de retração e fluência. Retração é a redução de volume de um elemento de concreto que independe de um carregamento externo aplicado, e pode ser classificada como retração hidráulica, química, autógena e térmica. A retração hidráulica ocorre pela perda de água do concreto para o meio ambiente, sendo diretamente influenciada pelas condições ambientais (umidade relativa, temperatura, vento, etc). Já a retração química ocorre pela diminuição de volume dos produtos gerados pela hidratação do cimento em comparação aos materiais que deram origem à reação. A retração autógena se dá a partir da perda de água consumida na hidratação do cimento. Por fim, a retração térmica é provocada pelo calor liberado na reação de hidratação que, em um primeiro momento, expande o 
concreto, mas apresenta uma redução de volume ao se resfriar. Os efeitos de retração considerados no modelo serão baseados no CEB-FIP Model Code 90 [7].

A fluência é caracterizada pela deformação contínua que ocorre quando um elemento de concreto é submetido a tensões constantes ao longo do tempo. Segundo Dias (2013) [8], a eficiência de uma análise numérica de fluência em estruturas requer a conversão da lei de fluência do tipo integral para uma lei em termos de taxa. Aparentemente isso é sempre possível, não de forma exata, porém com uma boa aproximação. Desta forma, neste trabalho será utilizada a teoria da solidificação proposta por Bazant e Prasannan (1989) [9], tendo alguns parâmetros ajustados através do CEBFIP Model Code 90 [7].

O comportamento viscoelástico de um material pode ser representado através do emprego de modelos reológicos formados por elementos de mola e de amortecedor. As associações mais simples consistem em uma mola e um amortecedor em série (Modelo de Maxwell) ou em paralelo (Modelo de Kelvin), conforme mostrado na Figura 1.

(a)

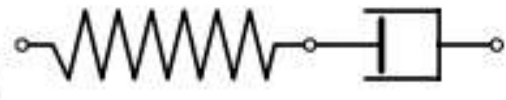

(b)

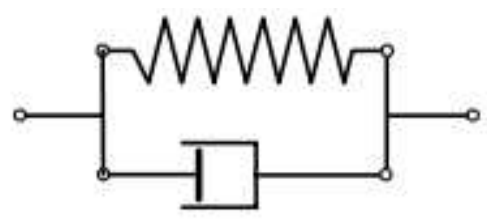

Figura 1: (a) Modelo de Maxwell; (b) Modelo de Kelvin.

Para um segmento de mola a relação entre tensão e deformação é dada pela Lei de Hooke, conforme a Equação (1).

$$
\sigma(t)=E . \varepsilon(t)
$$

onde: $\sigma(t)$ e $\varepsilon(t)$ representam a tensão e a deformação no instante $t$, respectivamente, e $E$ é o módulo de elasticidade.

Já o comportamento do amortecedor é representado pela Equação (2).

$$
\sigma(t)=\eta \cdot \dot{\varepsilon}(t)=\eta \frac{d \varepsilon}{d t}
$$

onde: $\eta$ é o coeficiente de viscoelasticidade e $\dot{\varepsilon}$ é a taxa de deformação.

As equações de equilíbrio e compatibilidade e as relações constitutivas para os dois modelos são apresentas na Tabela 1, sendo os índices $E$ e $\eta$ referentes as tensões ou deformações na mola e no amortecedor, respectivamente.

Tabela 1: Equações e relações dos modelos de Maxwell e Kelvin.

\begin{tabular}{ccc}
\hline & Modelo de Maxwell & Modelo de Kelvin \\
\hline Equação de equilíbrio & $\sigma(t)=\sigma_{E}(t)=\sigma_{\eta}(t)$ & $\sigma(t)=\sigma_{E}(t)+\sigma_{\eta}(t)$ \\
Equação de compatibilidade & $\varepsilon(t)=\varepsilon_{E}(t)+\varepsilon_{\eta}(t)$ & $\varepsilon(t)=\varepsilon_{E}(t)=\varepsilon_{\eta}(t)$ \\
Relação constitutiva da mola & $\sigma_{E}(t)=E \cdot \varepsilon_{E}(t)$ & $\sigma_{E}(t)=E \cdot \varepsilon_{E}(t)$ \\
Relação constitutiva do amortecedor & $\sigma_{\eta}(t)=\eta \cdot \dot{\varepsilon}_{\eta}(t)$ & $\sigma_{\eta}(t)=\eta \cdot \dot{\varepsilon}_{\eta}(t)$ \\
\hline
\end{tabular}

Uma melhor aproximação do comportamento viscoelástico pode ser obtida empregando modelos combinados com um grande número de molas e amortecedores em série ou em paralelo. Com isso pode-se obter diversos tempos de retardação, o que é característico de materiais complexos como o concreto [8]. A escolha por um modelo implica em diferentes equações de equilíbrio e compatibilidade, que levam a diferentes formulações para as tensões e deformações. O modelo 
adotado neste trabalho, e representado na Figura 2, é o modelo de Kelvin composto por até seis unidades. Este modelo foi empregado também em outros estudos $[8,11,12,13]$.

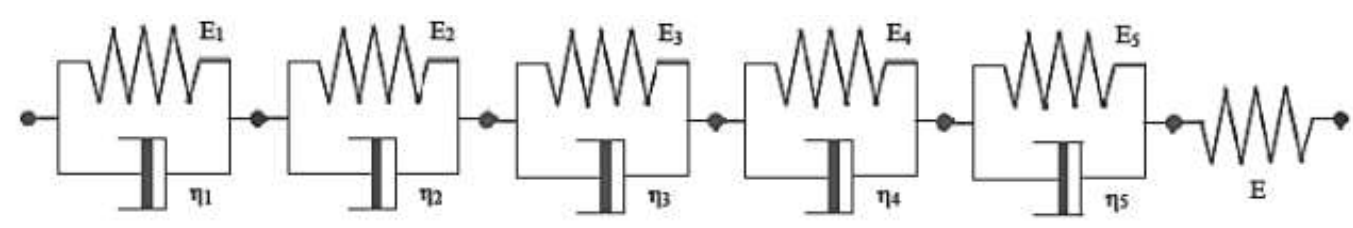

Figura 2: Representação do Modelo de Kelvin [12].

As tensões observadas em todas as camadas são as mesmas e a deformação resultante é dada pelo somatório das deformações em cada camada, conforme apresentado nas Equações (3) e (4).

$$
\begin{aligned}
& \varepsilon\left(t^{\prime}\right)=\varepsilon_{0}\left(t^{\prime}\right)+\sum_{\mu=1}^{5} \varepsilon_{\mu}\left(t^{\prime}\right) \\
& \dot{\sigma}\left(t^{\prime}\right)=\dot{\varepsilon}_{\mu}\left(t^{\prime}\right)\left\{E_{\mu}\left(t^{\prime}\right)+\dot{\eta}_{\mu}\left(t^{\prime}\right)\right\}+\eta_{\mu}\left(t^{\prime}\right) \ddot{\varepsilon}_{\mu}\left(t^{\prime}\right)
\end{aligned}
$$

onde: $\varepsilon\left(t^{\prime}\right)$ é a deformação total; $\varepsilon_{0}\left(t^{\prime}\right)$ é a deformação na mola isolada; $\varepsilon_{\mu}\left(t^{\prime}\right)$ é a deformação na camada $\mu ; \dot{\sigma}\left(t^{\prime}\right)$ é a primeira derivada em relação ao tempo da tensão total atuante na cadeia de Kelvin; $\dot{\varepsilon}_{\mu}\left(t^{\prime}\right)$ é a primeira derivada no tempo da deformação da unidade $\mu ; E_{\mu}\left(t^{\prime}\right)$ é o módulo de elasticidade da mola $\mu ; \eta_{\mu}\left(t^{\prime}\right)$ é o coeficiente de viscosidade do amortecedor $\mu$; e $\ddot{\varepsilon}_{\mu}\left(t^{\prime}\right)$ é a segunda derivada no tempo da deformação da unidade $\mu$. Todos os parâmetros citados são dependentes da idade $t$ ' do concreto.

Esta formulação conduz a uma equação diferencial de segunda ordem, por isso por muito tempo preferiu-se utilizar a formulação de Maxwell que leva a uma equação diferencial de primeira ordem. Entretanto, a formulação de Bazant e Prasannan (1989) [9] apresentou a teoria da solidificação para a fluência, o que trouxe um novo uso para a cadeia de Kelvin [8].

Segundo Bazant e Prasannan (1989) [9] a teoria da solidificação afirma que o efeito do envelhecimento relacionado à fluência do concreto é devido à alteração do volume de concreto solidificado ao longo do tempo. Dessa forma, o envelhecimento do concreto é expresso por uma função $V(t)$, enquanto as outras propriedades do concreto não dependem da sua idade, ou seja, se mantém constantes ao longo do tempo. Assim a formulação de Kelvin para a tensão apresentada na Equação (4) fica simplificada conforme apresentado pela Equação (5).

$$
\sigma=E_{\mu} \varepsilon_{\mu}+\eta_{\mu} \dot{\varepsilon}_{\mu}
$$

onde: $\sigma$ é a tensão atuante; $E_{\mu}$ é o módulo de elasticidade da mola $\mu$, independente da idade do concreto; $\varepsilon_{\mu}$ é a deformação na camada $\mu ; \eta_{\mu}$ é o coeficiente de viscosidade do amortecedor $\mu$, independente da idade do concreto; e $\dot{\varepsilon}_{\mu}$ é a derivada no tempo da deformação na camada $\mu$.

Integrando a equação diferencial apresentada, obtém-se a expressão que define a deformação viscosa resultante da cadeia de Kelvin, conforme a Equação (6). A partir disso, obtém-se diretamente a função de fluência conforme a Equação (7).

$$
\varepsilon_{c}\left(t, t^{\prime}\right)=\sigma\left(t^{\prime}\right) \cdot \sum_{\mu=1}^{5} \frac{1}{E_{\mu}}\left(1-e^{-\left(t-t^{\prime}\right) / \tau_{\mu}}\right)
$$




$$
\gamma\left(t, t^{\prime}\right)=\sum_{\mu=1}^{5} \frac{1}{E_{\mu}}\left(1-e^{-\left(t-t^{\prime}\right) / \tau_{\mu}}\right) ; \quad \tau_{\mu}=\frac{\eta_{\mu}}{E_{\mu}}
$$

onde: $\varepsilon_{c}\left(t, t^{\prime}\right)$ é a deformação da cadeia de Kelvin sem envelhecimento; $\sigma\left(t^{\prime}\right)$ é a tensão atuante, aplicada na idade $t^{\prime}$ do concreto; $\gamma\left(t, t^{\prime}\right)$ é a função de fluência sem envelhecimento; $t$ ' é a idade do concreto quando foi aplicada a carga; $t$ é a idade do concreto e $\tau_{\mu}$ é o tempo de retardamento da unidade $\mu$.

Segundo Bazant e Prasannan (1989) [9], a deformação total no concreto é dada pela soma das deformações devido às partes elástica, viscoelástica, à temperatura, retração e fissuração, conforme mostrado na Figura 3.

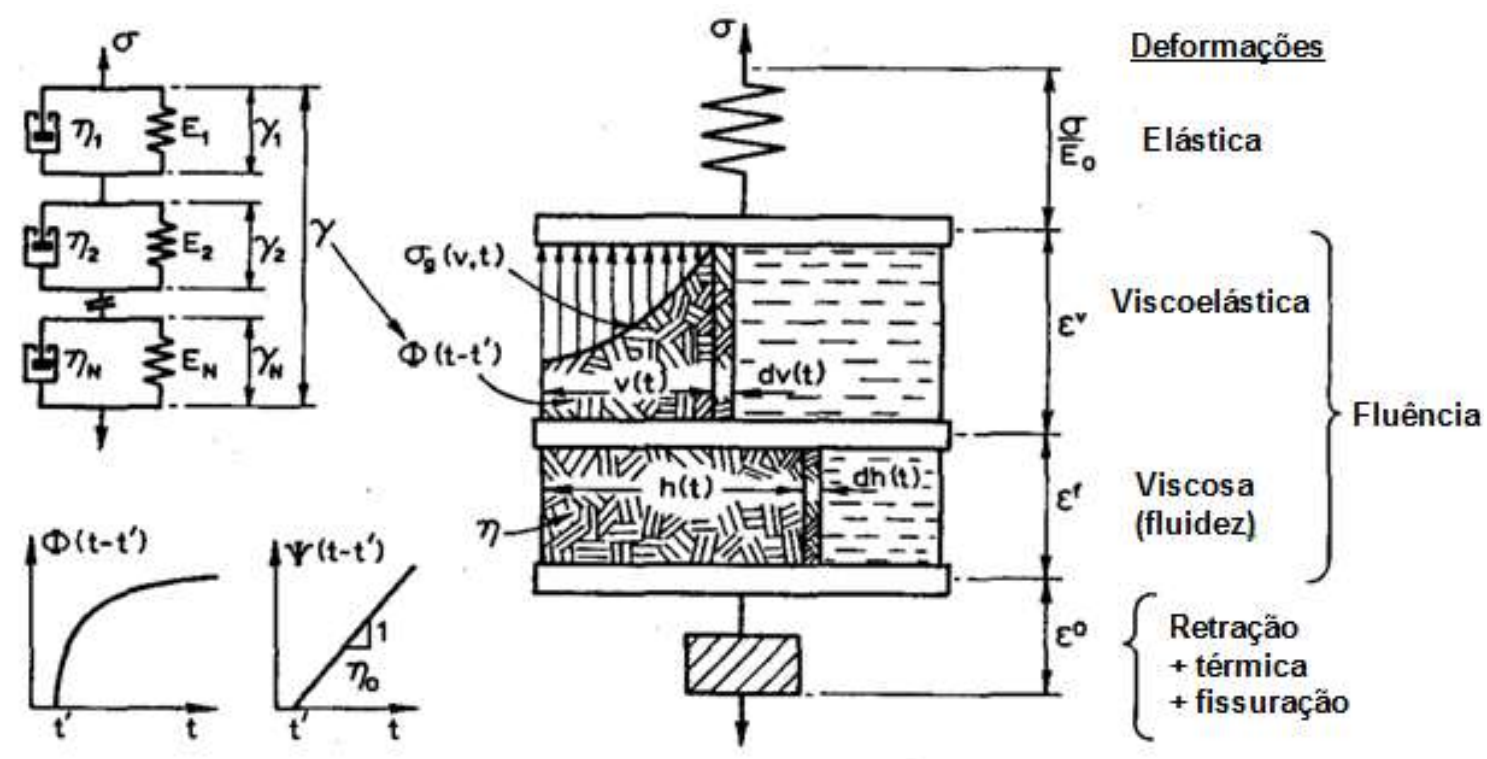

Figura 3: Representação do concreto segundo Bazant e Prasannan (1989) [9].

O modelo para fluência dado pelo CEB-FIP Model Code 90 [7] é válido para casos onde a tensão no concreto seja inferior a $40 \%$ da resistência à compressão média na idade de carregamento. Assim, as parcelas referentes à deformação viscoelástica $\left(\varepsilon^{v}\right)$ e à deformação viscosa $\left(\varepsilon^{f}\right)$, foram consideradas juntas na lei de fluência. A deformação total do concreto é dada pela Equação (8).

$$
\varepsilon\left(t, t^{\prime}\right)=\frac{\sigma}{E\left(t^{\prime}\right)}+\varepsilon_{c}\left(t, t^{\prime}\right)+\varepsilon_{0}(t)
$$

onde: $E\left(t^{\prime}\right)$ é o módulo de elasticidade da mola isolada para idade do concreto $t^{\prime} ; \varepsilon_{c}\left(t, t^{\prime}\right)$ é a deformação devida à fluência e $\varepsilon_{0}(t)$ é a deformação devida à retração.

A partir da teoria da solidificação, a expressão para a função de fluência pode ser dada pela Equação (9).

$$
J\left(t, t^{\prime}\right)=\frac{1}{E\left(t^{\prime}\right)}+\frac{\gamma\left(t, t^{\prime}\right)}{V(t)}
$$

onde: $J\left(t, t^{\prime}\right)$ é a função de fluência considerando o envelhecimento e $V(t)$ é a função de envelhecimento devida à variação do volume do concreto.

Para definição dos módulos de elasticidade da cadeia de Kelvin, é preciso aproximar a função $\gamma\left(t, t^{\prime}\right)$ apresentada na Equação (7), conhecida por série de Dirichlet (ou Prony), a um modelo de 
fluência escolhido, que é calculado em $N$ pontos. Essa aproximação é feita pelo método dos mínimos quadrados, que consiste na resolução do sistema: $[A]\{X\}=[B]$, sendo os componentes do sistema apresentados nas Equações (10), (11) e (12) [8]. É importante destacar que o ajuste do modelo pode levar a valores negativos de módulo de elasticidade, que não têm significado físico, sendo apenas um ajuste matemático [12].

$$
\begin{aligned}
& A(i, j)=\sum_{k=1}^{N}\left(1-e^{-\left(t-t^{\prime}\right) / \tau_{i}}\right)\left(1-e^{-\left(t-t^{\prime}\right) / \tau_{j}}\right) \\
& B(i)=\sum_{k=1}^{N} \gamma\left(t, t^{\prime}\right)\left(1-e^{-\left(t-t^{\prime}\right) / \tau_{i}}\right) \\
& X(j)=\frac{1}{E_{j}}
\end{aligned}
$$

A resolução de um sistema de equações está relacionada com o seu condicionamento e, para garantir que o sistema seja bem condicionado, devem-se definir os tempos de retardamento. Sugerese que os mesmos sigam o apresentado nas Equações (13), (14) e (15) [9].

$$
\begin{aligned}
& \tau_{\mu}=\tau_{1} \cdot 10^{\mu-1} ; \mu=1,2, \ldots, n \\
& \tau_{1}=0,01 t^{\prime} \\
& \tau_{N} \geq 0,5 t_{\text {max }}
\end{aligned}
$$

onde: $\tau_{1}$ é o tempo de retardamento para a unidade $1 ; t$ ' é o tempo de aplicação da primeira carga e $t_{\text {máx }}$ é o tempo final da análise.

Para resolução do sistema de equações apresentado anteriormente, se faz necessário definir os intervalos de tempo $\left(t-t^{\prime}\right)$. Bazant e Prasannan (1989) [9] sugerem que esses intervalos de tempo sejam lineares em escala logarítmica, conforme a Equação (16).

$$
\left(t-t^{\prime}\right)_{n}=10^{1 / m} \cdot\left(t-t^{\prime}\right)_{n-1} ; n=1,2, \ldots
$$

onde: $m$ é o número de intervalos de tempo em uma década.

Com um valor de $m$ igual a 10 já se tem uma boa aproximação, e o primeiro passo de tempo pode ser definido conforme a Equação (17) [8].

$$
\left(t-t^{\prime}\right)_{1}=0,1 t^{\prime}
$$

A resolução da Equação (4) se dá a partir de uma integração numérica e, para que esta seja mais efetiva, utiliza-se o chamado algoritmo exponencial, de forma que os passos de tempo podem chegar a valores bem maiores que o menor dos tempos de retardação. Nesse algoritmo considerase que a tensão varia linearmente com o tempo. Assim, a solução da Equação (5) é expressa nas Equações (18), (19) e (20) [9].

$$
\begin{aligned}
& \varepsilon_{\mu(i+1)}=\varepsilon_{\mu(i)} e^{-\Delta y_{\mu}}+\frac{\sigma_{i}}{E_{\mu}}\left(1-e^{-\Delta y_{\mu}}\right)+\frac{1-\lambda_{\mu}}{E_{\mu}} \Delta \sigma \\
& \Delta y_{\mu}=\frac{\Delta t}{\tau_{\mu}}
\end{aligned}
$$




$$
\lambda_{\mu}=\frac{\left(1-e^{-\Delta y_{\mu}}\right)}{\Delta y_{\mu}}
$$

onde: $\varepsilon_{\mu(i+1)}$ é a deformação viscoelástica da unidade $\mu$ sem envelhecimento, para o tempo $t_{i+1}$; $\varepsilon_{\mu(i)}$ é a deformação viscoelástica da unidade $\mu$ sem envelhecimento, para o tempo $t_{i}$; $\sigma_{i}$ é a tensão aplicada no tempo $t_{i} ; E_{\mu}$ é o módulo de elasticidade da mola $\mu ; \Delta t$ é a variação de tempo entre $t_{i+1} \mathrm{e}$ $t_{i}$; $\tau_{\mu}$ é o tempo de retardamento da unidade $\mu ; \Delta y_{\mu}$ e $\lambda_{\mu}$ são variáveis auxiliares no cálculo da deformação viscoelástica.

Já o incremento de deformação viscoelástica sem envelhecimento pode ser calculado como mostrado na Equação (21).

$$
\Delta \varepsilon_{\mu}=\varepsilon_{\mu(i+1)}-\varepsilon_{\mu(i)}
$$

Logo, substituindo a Equação (18) na Equação (21), tem-se:

$$
\begin{aligned}
& \Delta \varepsilon_{\mu}=\left(\frac{\sigma_{i}}{E_{\mu}}-\varepsilon_{\mu(i)}\right) \cdot\left(1-e^{-\Delta y_{\mu}}\right)+\frac{1-\lambda_{\mu}}{E_{\mu}} \cdot \Delta \sigma \\
& \varepsilon_{\mu(i)}^{*}=\frac{\sigma_{i}}{E_{\mu}}-\varepsilon_{\mu(i)}
\end{aligned}
$$

onde: $\varepsilon_{\mu}^{*}$ é a deformação viscosa da unidade $\mu$.

Mas, o que de fato interessa no processo iterativo da solução, é definir a parcela viscosa e não a deformação total. Assim, a partir da Equação (23), pode-se escrever a Equação (24).

$$
\varepsilon_{\mu(i+1)}^{*}=\frac{\sigma_{i+1}}{E_{\mu}}-\varepsilon_{\mu(i+1)}
$$

Conforme deduzido por Dias (2013) [8], substituindo a Equação (18) na Equação (24), pode-se calcular a deformação em cada unidade de Kelvin conforme a Equação (25).

$$
\varepsilon_{\mu(i+1)}^{*}=\frac{\lambda_{\mu}}{E_{\mu}} \cdot \Delta \sigma+\varepsilon_{\mu(i)}^{*} \cdot e^{-\Delta y_{\mu}}
$$

onde: $\Delta \sigma$ é a variação de tensão no dado intervalo.

Ao se introduzir o envelhecimento a partir da função de mudança de volume $V(t)$, obtém-se a Equação (26).

$$
\varepsilon_{\mu(i+1)}^{*}=\frac{\lambda_{\mu}}{E_{\mu} V_{i+\frac{1}{2}}} \cdot \Delta \sigma+\varepsilon_{\mu(i)}^{*} \cdot e^{-\Delta y_{\mu}}
$$

onde: $V_{i+1 / 2}$ é o volume do concreto calculado para o meio do intervalo de tempo entre $t_{i}$ e $t_{i+1}$.

Logo, o incremento de deformação viscosa total da cadeia de Kelvin, considerando o envelhecimento, é dado pela Equação (27) e de forma que $\Delta \varepsilon^{*}$ é o incremento de deformação não elástica, pois foi acrescentada a parcela devido à retração $\Delta \varepsilon_{0}$ que é independente da tensão. 


$$
\Delta \varepsilon^{*}=\sum_{\mu=1}^{N} \varepsilon_{\mu(i)}^{*} \cdot\left(1-e^{-\Delta y_{\mu}}\right)+\Delta \varepsilon_{0}
$$

Foi utilizado o modelo de fluência apresentado pelo CEB-FIP Model Code 90 [7] para a determinação da função dada na Equação (9), a partir do ajuste dos módulos de elasticidade das unidades de Kelvin pelo método dos mínimos quadrados e pela definição dos valores que representam a função $V(t)$ [12]. Este modelo, representado na Equação (28), é limitado a concretos com resistência entre 1,2 e $8 \mathrm{kN} / \mathrm{cm}^{2}(12$ a $80 \mathrm{MPa})$, umidades do ar entre 40 e $100 \%$ e tensões no concreto que não podem superar $40 \%$ da sua resistência média à compressão calculada para o momento de aplicação da carga.

$$
J\left(t, t^{\prime}\right)=\frac{1}{E\left(t^{\prime}\right)}+\frac{\phi\left(t, t^{\prime}\right)}{E_{c i}}
$$

onde: $E_{c i}$ é o módulo de elasticidade do concreto na idade de 28 dias e $\phi\left(t, t^{\prime}\right)$ é o coeficiente de fluência dado pela Equação (29).

$$
\phi\left(t, t^{\prime}\right)=\phi_{0} \beta_{c}\left(t, t^{\prime}\right)
$$

onde: $\phi_{0}$ é o coeficiente de fluência devido à idade de aplicação da carga e $\beta_{c}\left(t, t^{\prime}\right)$ é o coeficiente que descreve a fluência devido ao tempo de aplicação da carga.

Comparando a Equação (9) e a Equação (28) pode-se definir o coeficiente de fluência sem envelhecimento, que será ajustado pela cadeia de Kelvin, e a função $V(t)$ responsável pelo envelhecimento, conforme as Equações (30) e (31).

$$
\begin{aligned}
& \gamma\left(t, t^{\prime}\right)=\beta_{c}\left(t, t^{\prime}\right) \\
& V(t)=\frac{E_{c i}}{\phi_{0}}
\end{aligned}
$$

O coeficiente de fluência $\phi_{0}$, que depende da idade de aplicação da carga, é calculado a partir da Equação (32).

$$
\phi_{0}=\phi_{R H} \beta\left(f_{c m}\right) \beta\left(t^{\prime}\right)
$$

onde: $\phi_{R H}$ é o coeficiente devido à umidade do ambiente; $\beta\left(f_{c m}\right)$ é o coeficiente devido à resistência média à compressão do concreto e $\beta\left(t^{\prime}\right)$ é o coeficiente devido à idade do concreto na aplicação da carga.

O coeficiente relacionado à umidade do ambiente é calculado conforme a Equação (33), e para que se considere o efeito de temperaturas diferentes de $20^{\circ} \mathrm{C}$, deve ser corrigido conforme apresentado nas Equações (34) e (35).

$$
\begin{aligned}
& \phi_{R H}=1+\frac{1-R H / 100}{0,46(h / 100)^{1 / 3}} \\
& \phi_{R H, T}=\phi_{T}+\left(\phi_{R H}-1\right) \cdot \phi_{T}^{1,2} \\
& \phi_{T}=e^{0,015 .(T-20)}
\end{aligned}
$$

onde: $R H$ é a umidade do ambiente (\%); $h$ é a espessura fictícia da peça de concreto em milímetro, dada pela área da seção transversal dividido pelo perímetro da seção que está exposto à atmosfera; 
$\phi_{T}$ é o fator que leva em consideração temperaturas diferentes de $20^{\circ} \mathrm{C} ; \phi_{R H, T}$ é o fator $\phi_{R H}$ corrigido pelo efeito de temperatura e $T$ é a temperatura $\mathrm{em}^{\circ} \mathrm{C}$.

Os coeficientes $\beta\left(f_{c m}\right)$ e $\beta\left(t^{\prime}\right)$ são calculados a partir das Equações (36) e (37).

$$
\begin{aligned}
& \beta\left(f_{c m}\right)=\frac{5,3}{f_{c m 28} 0,5} \\
& \beta\left(t^{\prime}\right)=\frac{1}{0,1+\left(t_{a j}^{\prime}\right)^{0,2}}
\end{aligned}
$$

onde: $f_{c m 28}$ é a resistência média à compressão uniaxial do concreto aos 28 dias, neste caso em MPa e $t^{\prime}{ }_{a j}$ é a idade do concreto quando foi aplicada a carga ajustada devido ao efeito da temperatura e ao tipo de cimento, e determinada através da Equação (38).

$$
t_{a j}^{\prime}=t_{T}^{\prime} \cdot\left[\frac{9}{2+\left(t_{T}^{\prime}\right)^{1,2}}+1\right]^{\alpha} \geq 0,5
$$

onde: $t_{T}^{\prime}$ é a idade do concreto quando foi aplicada a carga ajustada devido ao efeito da temperatura, calculada através da Equação (39) e $\alpha$ é o fator relacionado com o tipo de cimento sendo, -1 para endurecimento lento, 0 para endurecimento normal a rápido e 1 para endurecimento rápido e cimento de alta resistência.

$$
t_{T}^{\prime}=\sum_{i=1}^{N} \Delta t_{i} \cdot \exp \left[13,65-\frac{4000}{273+T_{i}\left(\Delta t_{i}\right)}\right]
$$

onde: $\Delta t_{i}$ é o número de dias em que a temperatura se manteve igual a $T_{i}$ e $T_{i}\left(\Delta t_{i}\right)$ é a temperatura, em ${ }^{\circ} \mathrm{C}$.

O coeficiente $\beta_{c}\left(t, t^{\prime}\right)$, que descreve a fluência devido ao tempo de aplicação da carga e que está presente na Equação (29), pode ser calculado pela Equação (40).

$$
\beta_{c}\left(t, t^{\prime}\right)=\left[\frac{\left(t-t^{\prime}\right)}{\beta_{H}+\left(t-t^{\prime}\right)}\right]^{0,3}
$$

onde: $\beta_{H}$ é o fator relacionado a umidade do ar, e pode ser determinado através da Equação (41).

$$
\beta_{H}\left(t, t^{\prime}\right)=150 .\left[1+\left(1,2 \frac{R H}{100}\right)^{18}\right] \cdot \frac{h}{100}+250 \leq 1500
$$
(42).

Para considerar o efeito de temperatura, $\beta_{H}$ deve ser multiplicado por $\beta_{T}$, calculado pela Equação

$$
\beta_{T}=\exp \left(\frac{1500}{273+T}-5,12\right)
$$

onde: $\beta_{T}$ é o coeficiente que considera o efeito de temperaturas diferentes de $20^{\circ} \mathrm{Cem} \beta_{H}$.

Já em relação ao módulo de elasticidade para as diferentes idades do concreto, o CEB-FIP Model Code 90 [7] indica que este pode ser determinado a partir da Equação (43). 
$E\left(t^{\prime}\right)=\beta_{E}(t) \cdot E_{c i}$

onde: $E\left(t^{\prime}\right)$ é o módulo de elasticidade da mola isolada para idade do concreto $t^{\prime}$ e $\beta_{E}(t)$ é o fator de correção dependente da idade do concreto, que pode ser determinada pela Equação (44).

$$
\beta_{E}(t)=\sqrt{\beta_{c c}(t)}
$$

onde: $\beta_{c c}(t)$ é o fator de correção dependente da idade do concreto, podendo ser determinado pela Equação (45).

$$
\beta_{c c}(t)=\exp \left\{s .\left[1-\left(\frac{28}{t_{a j}^{\prime}}\right)^{0,5}\right]\right\}
$$

onde: $s$ é o fator dependente do tipo de cimento sendo, 0,38 para endurecimento lento, 0,25 para endurecimento normal a rápido e 0,20 para endurecimento rápido e cimento de alta resistência.

O módulo de elasticidade deve, também, ser ajustado devido ao efeito de temperatura diferente de $20^{\circ} \mathrm{C}$, conforme a Equação (46).

$$
E(T)=E\left(t^{\prime}\right) \cdot(1,06-0,003 T)
$$

Para o cálculo da deformação por retração o CEB-FIP Model Code 90 [7] indica a Equação (47).

$$
\varepsilon_{c s}\left(t, t_{s}\right)=\varepsilon_{c s 0} \beta_{s}\left(t, t_{s}\right)
$$

onde: $\varepsilon_{c s}\left(t, t_{s}\right)$ é a deformação por retração; $t_{s}$ é a idade do concreto em que inicia a retração, equivalente ao fim do período de cura úmida do concreto; $t$ é a idade do concreto; $\varepsilon_{c s o}$ é o coeficiente de retração básica e $\beta_{s}\left(t, t_{s}\right)$ é o coeficiente de retração devido ao tempo que o concreto está sofrendo retração.

O fator de retração básica $\varepsilon_{c s o}$ independe do tempo que o concreto está em cura úmida ou não e é calculado pela Equação (48).

$$
\varepsilon_{c s 0}=\varepsilon_{s}\left(f_{c m}\right) \cdot \beta_{R H}
$$

onde: $\varepsilon_{s}\left(f_{c m}\right)$ é o fator relacionado com a resistência média à compressão do concreto e $\beta_{R H}$ é o fator relacionado com a umidade do ambiente.

O coeficiente $\varepsilon_{s}\left(f_{c m}\right)$ pode ser calculado pela Equação (49).

$$
\varepsilon_{s}\left(f_{c m}\right)=\left[160+10 \beta_{s c}\left(9-f_{c m 28}\right)\right] \cdot 10^{-6}
$$

onde: $\beta_{s c}$ é o fator dependente do tipo de cimento, sendo 4 para endurecimento lento, 5 para endurecimento normal a rápido e 8 para endurecimento rápido e cimento de alta resistência.

Já o coeficiente $\beta_{R H}$ pode ser calculado pela Equação (50).

$$
\beta_{R H}=\left\{\begin{array}{c}
-1,55 \beta_{s R H} \rightarrow 40 \% \leq R H<99 \% \\
0,25 \rightarrow R H \geq 99 \%
\end{array}\right.
$$

onde: $\beta_{S R H}$ é o fator relacionado com a umidade do ambiente, podendo ser determinado a partir da Equação (51). 


$$
\beta_{\text {SRH }}=1-\left(\frac{R H}{100}\right)^{3}
$$

O coeficiente que representa a deformação devido ao tempo ao qual o concreto está sujeito aos efeitos de retração por secagem pode ser calculado pelas Equações (52) e (53).

$$
\begin{aligned}
& \beta_{s}\left(t, t_{s}\right)=\left[\frac{t-t_{s}}{\alpha_{s T}(T)+\left(t-t_{s}\right)}\right]^{0,5} \\
& \alpha_{s T}(T)=350 \cdot\left(\frac{h}{100}\right)^{2} \cdot \exp [-0,06(T-20)]
\end{aligned}
$$

\subsection{PARÂMETROS DOS ENSAIOS DE ROSS (1958)}

Ross (1958) [14] desenvolveu experimentos com diversas combinações de cargas e idades diferentes para o concreto, sendo, nestes ensaios, utilizados corpos de prova cilíndricos com 11,75 $\mathrm{cm}$ de diâmetro e 30,50 $\mathrm{cm}$ de altura. Cada plataforma de teste incorporou um dinamômetro de carga com possibilidade de medições de deformação ao longo de três comprimentos dispostos em intervalos de $120^{\circ}$ ao redor do perímetro. Não é mencionado no estudo se os corpos de prova foram selados para que não houvesse troca de umidade com o meio ambiente. Na Tabela 2 estão apresentadas as características principais do ensaio.

Tabela 2: Dados relativos ao ensaio de Ross (1958) [14].

\begin{tabular}{cccc}
\hline Parâmetro & Símbolo & Unidade & Valor \\
\hline Resistência característica à compressão (corpo de & $\mathrm{f}_{\mathrm{cm}, \text { cúb }}$ & $\mathrm{MPa}$ & 66,19 \\
prova cúbico) & $\mathrm{v}_{\mathrm{c}}$ & adimensional & 0,15 \\
Coeficiente de Poisson do concreto & $\mathrm{h}_{\mathrm{f}}$ & $\mathrm{cm}$ & 3,94 \\
Espessura fictícia & $\mathrm{t}_{\mathrm{s}}$ & dias & 7 \\
Idade do concreto no final da cura & $\mathrm{T}$ & ${ }^{\circ} \mathrm{C}$ & 17 \\
Temperatura & $\mathrm{RH}$ & ${ }^{\circ}$ & 93 \\
Umidade relativa do ambiente & $\mathrm{s}$ & adimensional & 0,2 \\
Coeficiente que depende do tipo de cimento & $\alpha$ & adimensional & 1 \\
Coeficiente que depende do tipo de cimento & $\beta_{\mathrm{sc}}$ & adimensional & 8 \\
Coeficiente que depende do tipo de cimento & & & \\
\hline
\end{tabular}

Foram utilizados corpos de prova cúbicos para a determinação da resistência à compressão do concreto $\left(f_{c m, c u ́ b}\right)$. Assim, baseado nas classes de resistência apresentadas no CEB-FIB Model Code (2010) [15] e seguindo o que foi utilizado por Westerberg (2008) [16], utilizou-se um fator de redução do valor da resistência de 0,8 para conversão dos valores relativos a corpos de prova cúbicos para cilíndricos.

Cada amostra carregada foi acompanhada por um corpo de prova de controle, ou seja, sem aplicação de carregamento, sob condições atmosféricas idênticas. Assim, o autor estimou as deformações por retração que apresentaram valores reduzidos devido à alta umidade do ar. As deformações por retração foram descontadas das totais medidas, a fim de trazer as deformações resultantes apenas relacionadas à tensão. Logo, os resultados apresentam a deformação instantânea elástica e a deformação devido à fluência. O histórico das tensões acumuladas no tempo, para cada

\begin{tabular}{|c|c|c|c|c|c|c|c|}
\hline Testes & $\mathbf{t}_{0}$ & $\mathbf{t}_{1}$ & $\mathbf{t}_{2}$ & $\mathbf{t}_{3}$ & $\mathbf{t}_{4}$ & $t_{5}$ & $\mathbf{t}_{\mathbf{f}}$ \\
\hline Teste 1 Idade [dias] & 14 & 60 & - & - & - & - & 140 \\
\hline
\end{tabular}
teste, é apresentado na Tabela 3.

Tabela 3: Histórico de tensões relativas ao ensaio de Ross (1958) [14]. 


\begin{tabular}{ccccccccc} 
& $\sigma[\mathrm{MPa}]$ & 15,03 & 0 & - & - & - & - & 0 \\
\hline \multirow{2}{*}{ Teste 2 } & Idade $[$ dias $]$ & 28 & 60 & 91 & 120 & 154 & - & 190 \\
& $\sigma[\mathrm{MPa}]$ & 15,03 & 11,27 & 7,51 & 3,76 & 0 & - & 0 \\
\hline \multirow{2}{*}{ Teste 3 } & Idade $[$ dias $]$ & 8 & 14 & 28 & 63 & 90 & 120 & 180 \\
& $\sigma[\mathrm{MPa}]$ & 13,79 & 11,103 & 8,27 & 5,51 & 2,75 & 0 & 0 \\
\hline \multirow{2}{*}{ Teste 4 } & Idade $[$ dias] & 8 & 16 & 28 & 63 & 90 & 120 & 180 \\
& $\sigma[\mathrm{MPa}]$ & 2,75 & 5,51 & 8,27 & 11,03 & 13,79 & 0 & 0 \\
\hline \multirow{2}{*}{ Teste 5 5} & Idade $[$ dias] & 8 & 14 & 28 & 63 & 90 & 120 & 180 \\
& $\sigma[\mathrm{MPa}]$ & 13,79 & 8,27 & 2,75 & 8,27 & 13,79 & 0 & 0 \\
\hline
\end{tabular}

\subsection{MODELAGEM COMPUTACIONAL}

Para a modelagem computacional foi utilizado o software ANSYS através da plataforma Mechanical APDL 15.0 associada à ferramenta UPF (User Programmable Features). Esta ferramenta corresponde a um conjunto de recursos nos quais o usuário pode intervir na análise numérica através de sub-rotinas externas que são integradas ao programa principal durante o processo de solução dos problemas em elementos finitos. Esta ferramenta permite, dentre outras possibilidades, a criação de um novo elemento ou a adaptação/implementação de um novo modelo constitutivo para os materiais empregados nas análises.

Neste trabalho é utilizada a sub-rotina USERMAT para a implementação do modelo constitutivo que representa o comportamento mecânico do concreto. A sub-rotina programada pelo usuário é "chamada" pelo programa principal durante o processo iterativo. A USERMAT possui internamente um conjunto de quatro sub-rotinas, que estão associadas ao tipo de elemento utilizado na análise, seja para elemento sólido (USERMAT3D), elemento de estado plano (USERMATPS), elementos de viga (USERMATBM) ou elementos de treliça (USERMAT1D). Dessa forma, a subrotina principal verifica o tipo de elemento adotado na análise e redireciona o processo de solução para a sub-rotina relacionada ao elemento, atualizando as tensões de acordo com o modelo constitutivo programado em linguagem FORTRAN. Por se tratar de um estudo acerca de um elemento linear a representação do modelo mecânico do concreto neste trabalho se dá através da USERMATBM.

No que tange ao elemento finito utilizado, para a modelagem do concreto foi utilizado o BEAM189. Este elemento é utilizado para análise linear ou não-linear de estruturas reticuladas, como pilares e vigas esbeltas ou moderadamente esbeltas, envolvendo grandes deslocamentos e diversos modelos mecânicos de materiais, tais como elasticidade, plasticidade e fluência. Trata-se de um elemento unidimensional contido no espaço tridimensional, o qual possui três nós ao longo de seu comprimento (formulação quadrática), e havendo seis graus de liberdade por nó, relativos às translações e rotações em tornos dos eixos locais x, y e z, conforme mostrado na Figura 4.

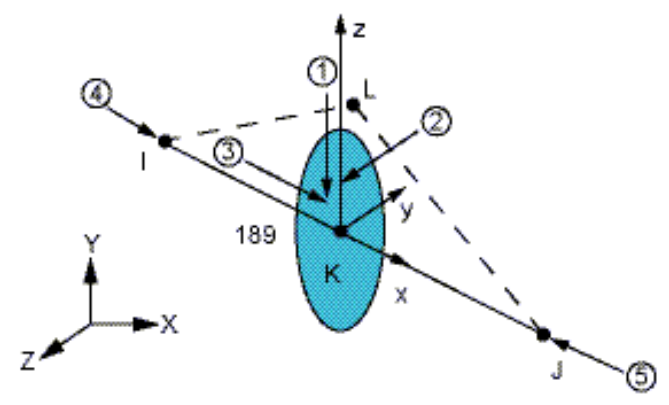

Figura 4: Geometria do elemento BEAM189 [17].

O ANSYS apresenta, para cada um de seus elementos, diversas KEYOPTS (opções chave) que permitem caracterizar o comportamento do elemento. Estas opções apresentam valores padrões, e estes devem ser verificados se são condizentes com a análise a ser realizada. Nesse sentido, este elemento possibilita a consideração de um grau de liberdade adicional, relativo ao empenamento, o qual por default não é considerado (idem neste trabalho), sendo necessário atribui-lo na KEYOPT(1) do elemento. Em relação às propriedades inerciais da seção transversal, estas podem 
ser alteradas na KEYOPT(2), o qual por padrão altera a área da seção transversal em função do alongamento axial, no sentido de preservar o volume da peça. As demais opções, de forma geral, estão relacionadas aos dados de saída que o usuário desejar obter, tais como tensões, deformações e esforços generalizados.

As propriedades dos materiais são fornecidas ao ANSYS através de scripts. Os comandos utilizados a fim de atribuir o material programado pelo usuário são TB,USER; TB,TEMP e TB,DATA. O primeiro informa ao programa o número de identificação do material, o número de temperaturas para as quais serão fornecidas as propriedades do material e o número de constantes que serão atribuídas a cada temperatura. O comando TB,TEMP informa para qual temperatura se está fornecendo os dados, e finalmente, TB,DATA é utilizado para atribuir as propriedades do material para a temperatura em questão. A Figura 5 ilustra um exemplo de script para inserção de dados para o material programado neste trabalho.

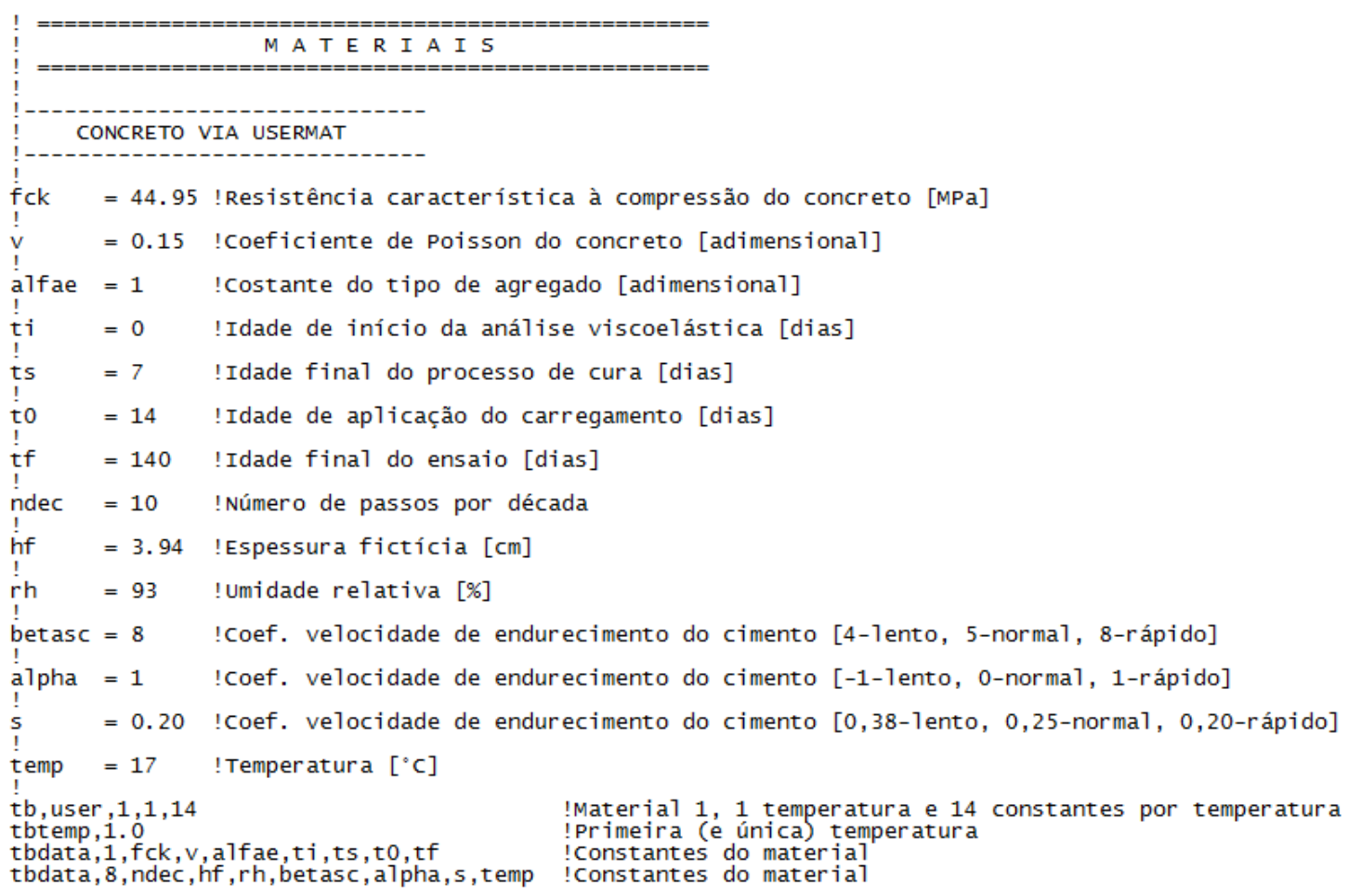

Figura 5: Exemplo de script para inserção de dados no ANSYS para o material programado pelo usuário.

As propriedades gerais e dimensões da seção transversal são definidas através dos comandos SECTYPE e SECDATA. Estes comandos definem o tipo de seção, sua forma e dimensões, bem como o refinamento da malha relativa à seção transversal, discretizada através do número de células ao longo da base e da altura da seção. O elemento conta com dois pontos de integração ao longo do seu comprimento, nos quais se avaliam tensões e deformações na seção transversal, e quatro pontos de integração por célula, conforme apresentado na Figura 6. Não sendo especificada uma malha à seção transversal, o padrão é a divisão em duas células ao longo de cada direção. 

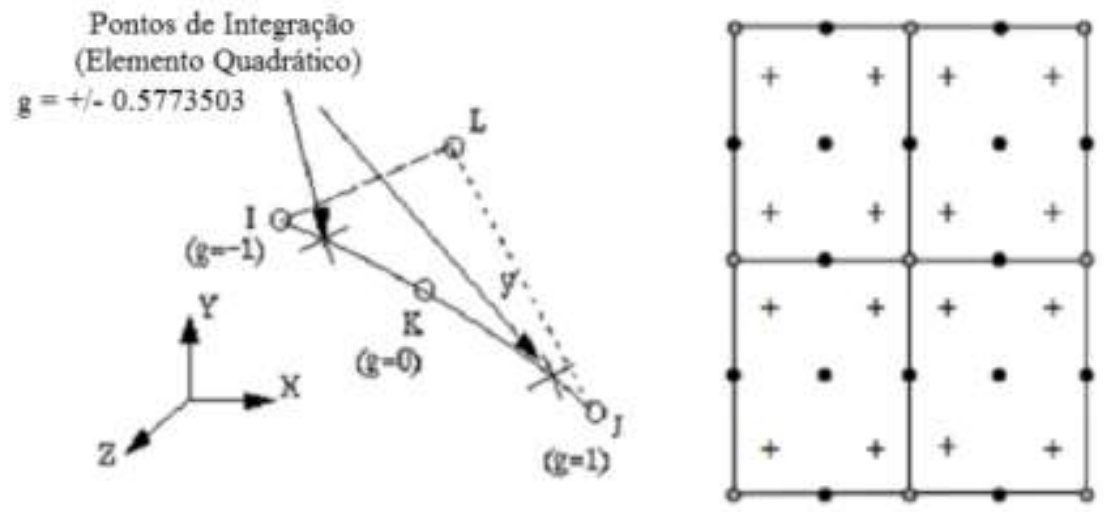

Figura 6: Pontos de integração e discretização da seção para o elemento BEAM189 [17].

A Figura 7 ilustra um exemplo de script para inserção de dados relativos à seção transversal dos corpos de prova de Ross (1958) [14]. Já a Figura 8 apresenta a modelagem final destes.

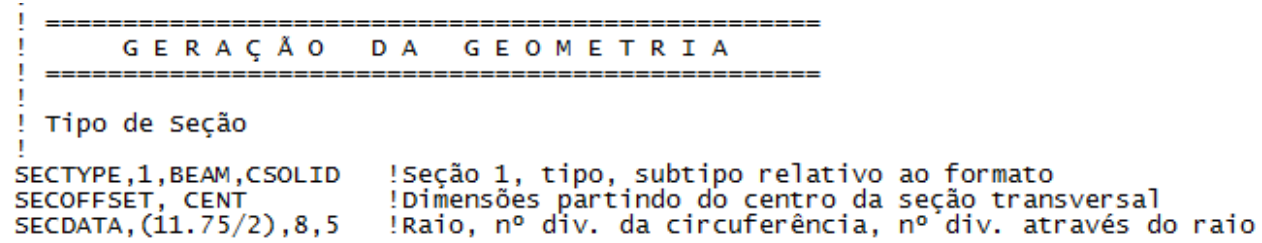

Figura 7: Script para inserção de dados da seção transversal dos corpos de prova de Ross (1958) [14].

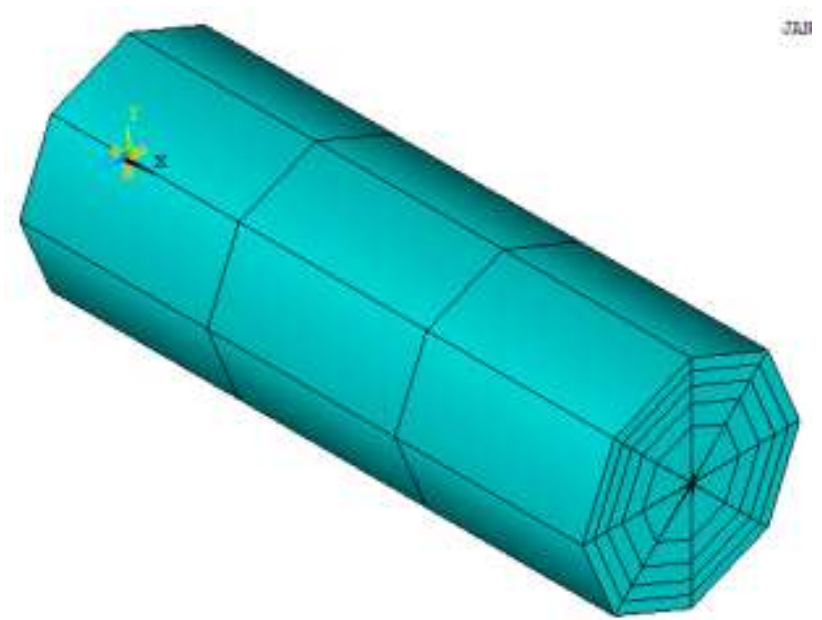

Figura 8: Modelagem dos elementos ensaiados por Ross (1958) [14].

\section{RESULTADOS E DISCUSSÃO}

Os resultados obtidos a partir do modelo proposto neste trabalho foram comparados com os resultados experimentais obtidos por Ross (1958) [14], e são apresentados nas Figuras 9 a 13. 


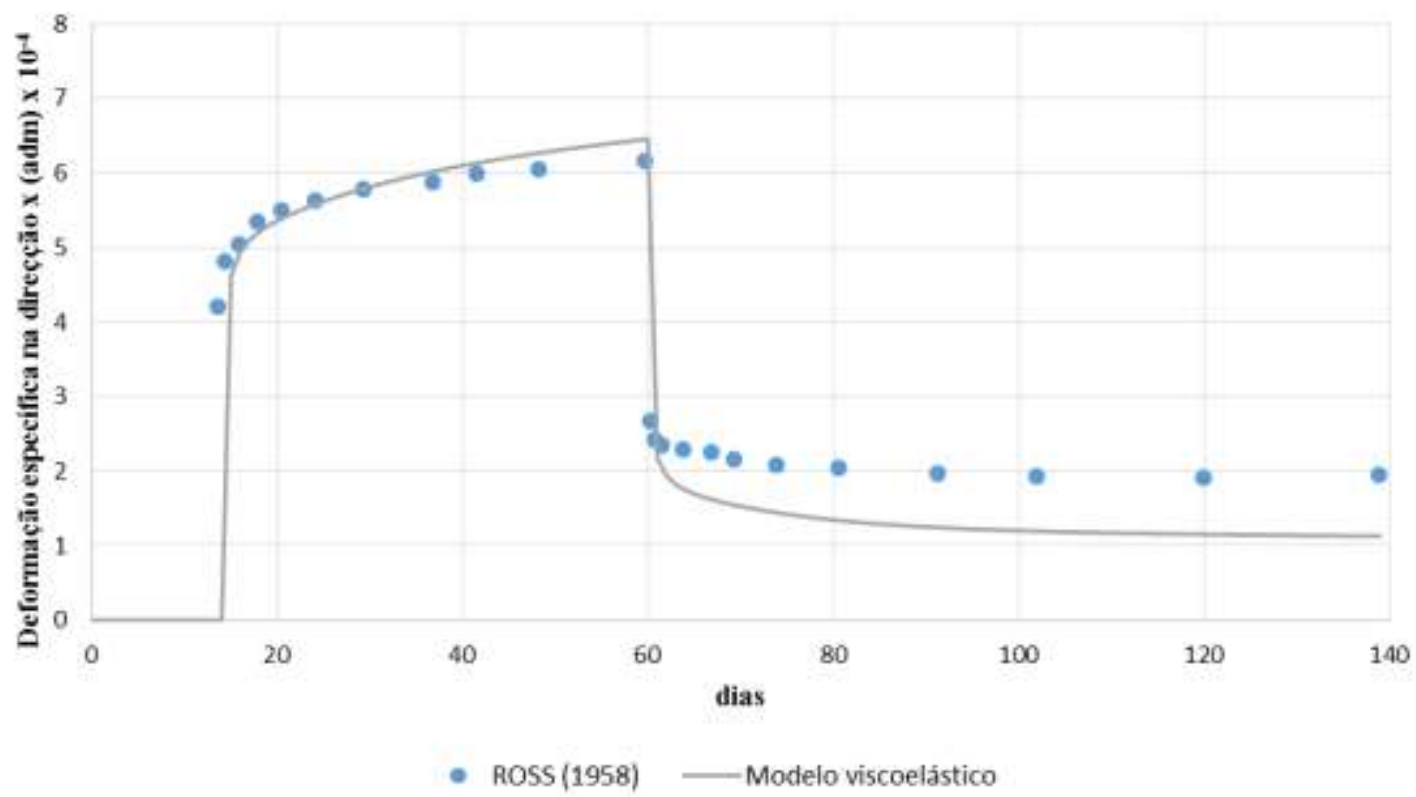

Figura 9: Comparação com os resultados obtidos por Ross (1958) [14] - Teste 1.

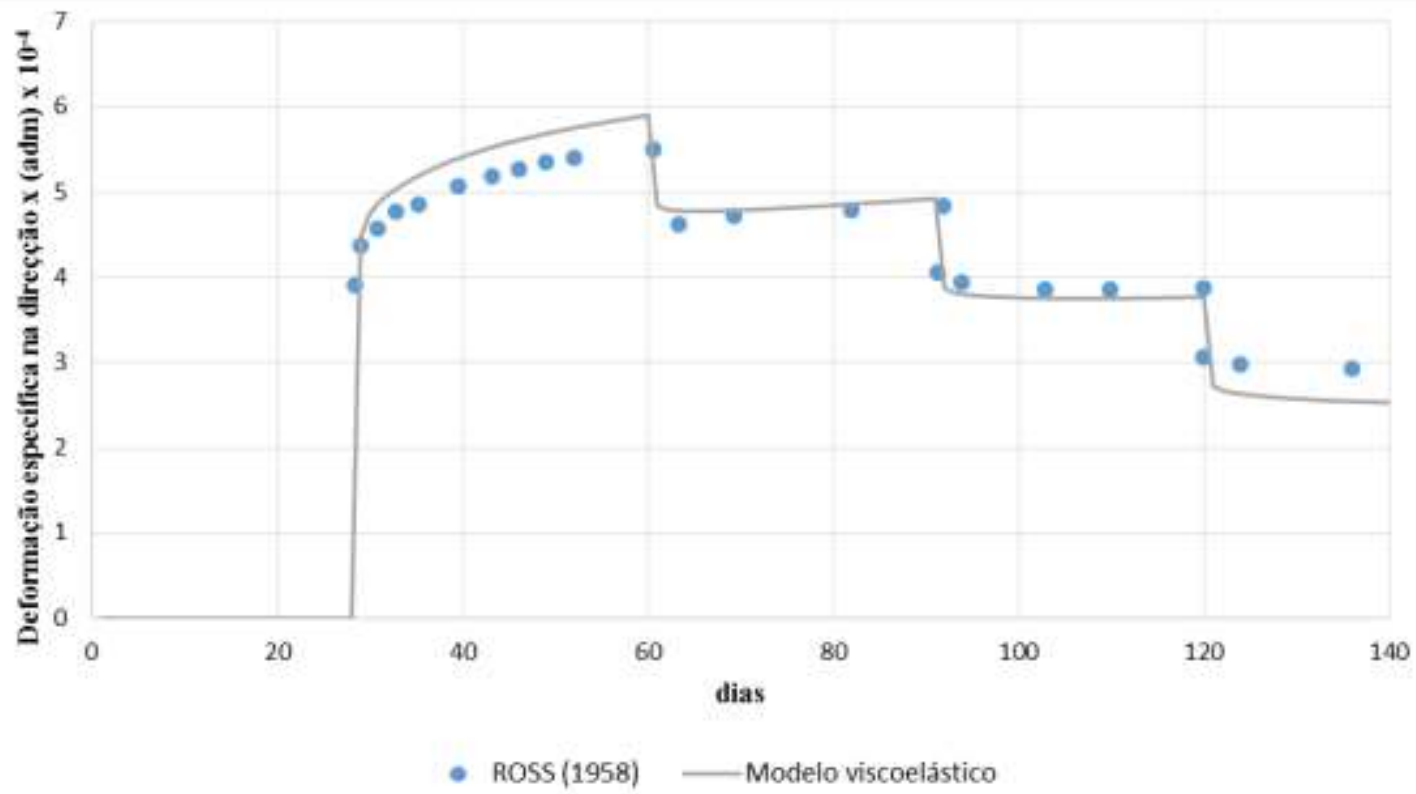

Figura 10: Comparação com os resultados obtidos por Ross (1958) [14] - Teste 2. 


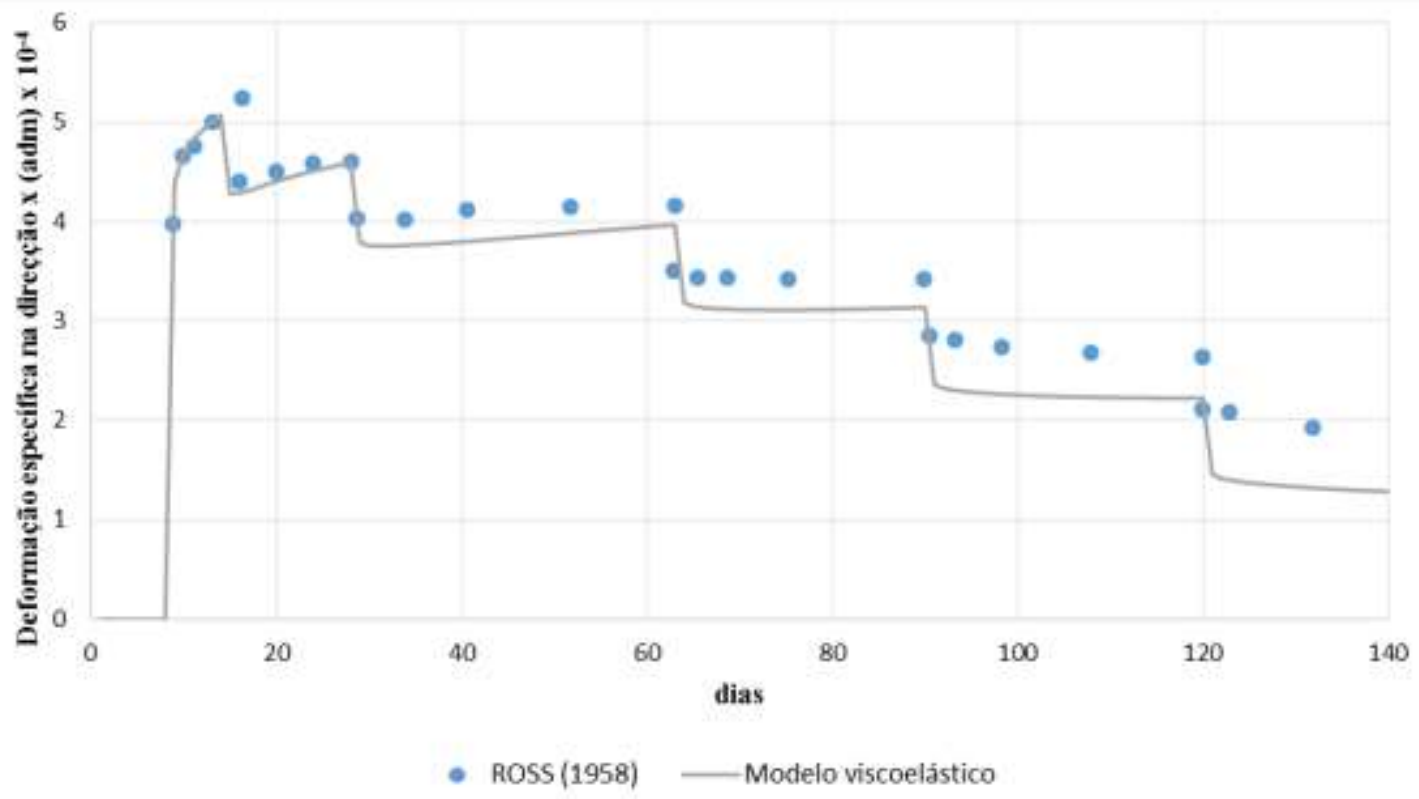

Figura 11: Comparação com os resultados obtidos por Ross (1958) [14] - Teste 3.

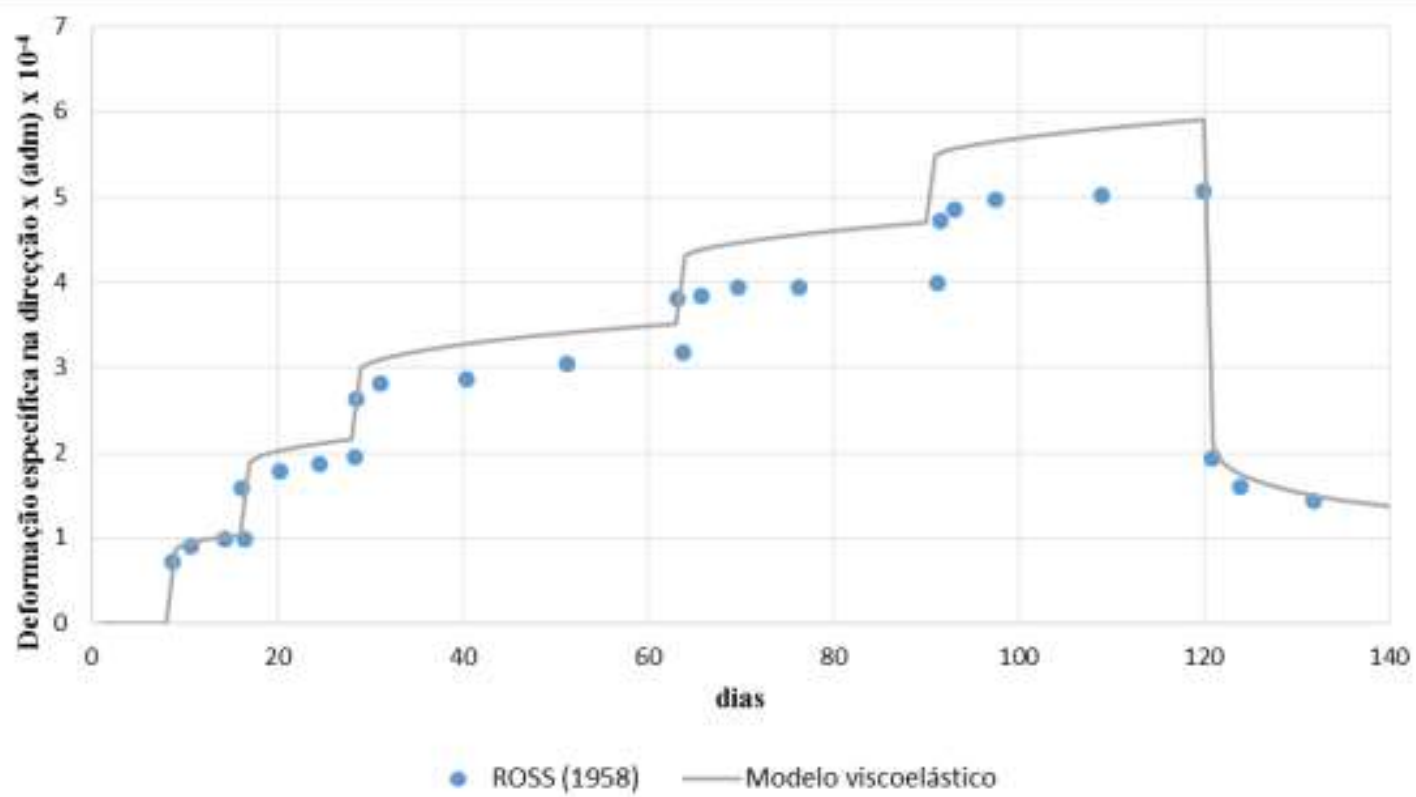

Figura 12: Comparação com os resultados obtidos por Ross (1958) [14] - Teste 4. 


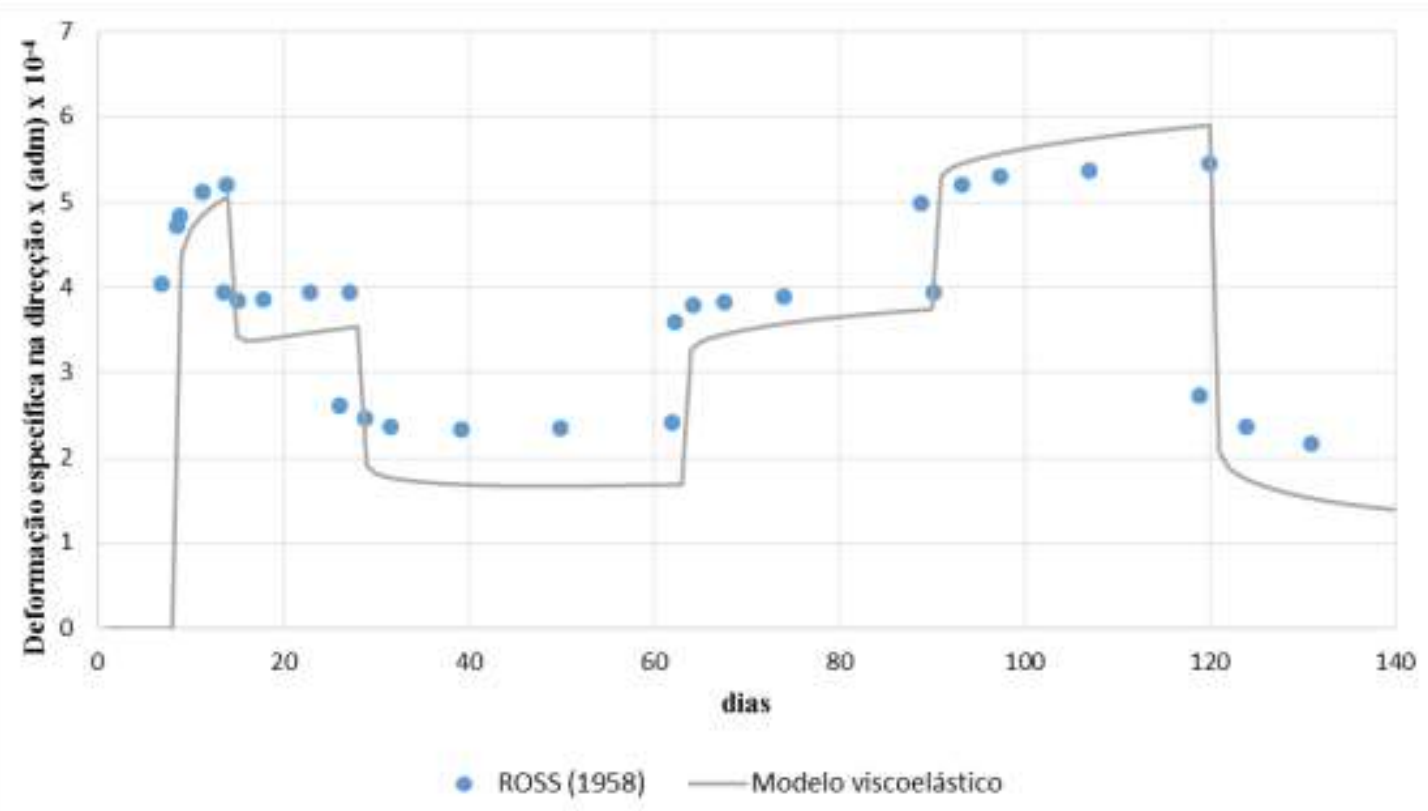

Figura 13: Comparação com os resultados obtidos por Ross (1958) [14] - Teste 5.

Notam-se nos resultados apresentados que os trechos de descarregamento apresentam diferenças mais acentuadas entre os resultados experimentais e os obtidos através do modelo viscoelástico. Na maioria dos ensaios, nos trechos de tensão descendente, as deformações acumuladas obtidas de maneira computacional se mostraram menores do que as deformações reais. Este comportamento se mostrou semelhante ao apresentado em Ross (1958) [14], quando a comparação dos resultados experimentais foi feita com resultados obtidos a partir do modelo da superposição. Já nos trechos de carregamento da estrutura, a comparação entre os resultados de deformação mostrou melhor ajuste, conforme mostrado na Tabela 4.

Tabela 4: Comparação entre as deformações obtidas experimentalmente e pelo modelo viscoelástico em pontos importantes de cada teste

\begin{tabular}{ccc}
\hline Teste & Tempo [dias] & $\boldsymbol{\varepsilon}_{\exp } / \boldsymbol{\varepsilon}_{\text {mod }}$ \\
\hline 1 & 60 & 0,95 \\
2 & 60 & 0,93 \\
3 & $14-16$ & 1,03 \\
4 & 120 & 0,86 \\
5 & 120 & 0,92 \\
\hline
\end{tabular}

Os pontos constantes na Tabela 4 foram selecionados em virtude de estarem situados em fases finais de carregamento que são mantidos constantes. A média e o desvio padrão dos valores de comparação da deformação específica foram 0,94 e 0,05, respectivamente. Analisando-se os resultados apresentados nas Figuras 9 a 13 e na Tabela 4, pode-se observar que o modelo customizado representa de maneira satisfatória os resultados experimentais obtidos por Ross (1958) [14], sobretudo quando o material é submetido a etapas de carregamento, comportamento este, condizente com o modelo de carregamento de estruturas usuais.

\section{CONCLUSÃO}

Os resultados aqui apresentados fazem parte de um estudo em andamento que visa determinar a confiabilidade de pilares de concreto armado submetidos a carregamentos de longa duração. Não impede, no entanto, que o modelo possa ser adaptado para outros tipos de estruturas, como para a previsão de flechas diferidas em vigas de concreto armado, por exemplo. Um estudo mais amplo 
se justifica na medida em que as estruturas de concreto, por terem suas dimensões cada vez menores devido à evolução das técnicas de projeto e da qualidade dos materiais, estão sujeitas a efeitos que antes se apresentavam menos importantes. Enquadram-se na situação citada os efeitos gerados por carregamentos de longa duração, aqui abordados.

Foi apresentado neste trabalho um modelo viscoelástico, e sua validação, para a representação do concreto levando-se em consideração os efeitos diferidos. A validação deste modelo visa a sua utilização, conjuntamente com um modelo elastoplástico, formando um modelo mais realista para análise de estruturas de concreto armado.

O modelo viscoelástico aqui apresentado, baseado na teoria da solidificação de Bazant e Prasannan (1989) [9] e ajustado pelo CEB-FIB Model Code 90 [7], foi implementado no software ANSYS através da rotina USERMAT e teve a verificação de sua validade a partir da comparação com resultados experimentais obtidos por Ross (1958) [14].

Os resultados obtidos mostraram-se satisfatórios no que diz respeito à representação dos efeitos diferidos do concreto, em especial para os trechos referentes à fase de carregamento da estrutura. Além dos resultados gráficos apresentados, foram definidos pontos considerados importantes em cada teste, normalmente em fase final de carregamento, para se quantificar o ajuste entre as deformações obtidas experimentalmente e as obtidas através do modelo numérico. A média da razão entre estas deformações $\left(\varepsilon_{\exp } / \varepsilon_{\text {mod }}\right.$ ), considerando todos os pontos determinados, foi de 0,94 , com um desvio padrão de 0,05 .

Por tudo que foi exposto, considera-se que os resultados obtidos neste estudo se mostraram adequados à representação de concretos submetidos a carregamentos de longa duração, sendo promissora a utilização deste modelo, em conjunto com um modelo elastoplástico, para análise de estruturas de concreto armado.

\section{AGRADECIMENTOS}

Os autores agradecem à FURG e ao IFRS - Campus Rio Grande, em especial do Programa de Pós-Graduação em Modelagem Computacional (PPGMC) e ao Laboratório de Estruturas e Materiais de Construção Civil (LEMCC), por darem condições para o desenvolvimento desta pesquisa. O segundo autor agradece o apoio do CNPq para o desenvolvimento desta pesquisa.

\section{REFERÊNCIAS BIBLIOGRÁFICAS}

1. Mehta PK, Monteiro PJM. Concreto - Microestrutura, Propriedades e Materiais. São Paulo: IBRACON; 2008. $674 \mathrm{p}$.

2. Barbosa AF, Ribeiro GO. Analysis of reinforced concrete structures using ANSYS nonlinear concrete model. In: Idelsohn S, Oñate E, Dvorkin E, editors. Proceedings of the 4th World Congress on Computational Mechanics; 1998 Jun 29 - Jul 2; Buenos Aires, AR. Barcelona (SP): CIMNE; 1998.

3. An G-H, Cha S-L, Kim J-K. Modification of the long-term deformation models for steel reinforced concrete columns. Constr Build Mater. 2018 Nov;189(1):245-52, doi:10.1016/j.conbuildmat.2018.08.095.

4. Chen P, Zheng W, Wang Y, Du K, Chang W. Strain recovery model for concrete after compressive creep. Constr Build Mater. 2019 Feb;199(1):746-55, doi:10.1016/j.conbuildmat.2018.12.085.

5. Ghasemzadeh F, Manafpour A, Sajedi S, Shekarchi M, Hatami M. Predicting long-term compressive creep of concrete using inverse analysis method. Constr Build Mater. 2016 Oct;124(1):496-507, doi:10.1016/j.conbuildmat.2016.06.137.

6. Jin S-S, Cha S-L, Jung H-J. Improvement of concrete creep prediction with probabilistic forecasting method under model uncertainty. Constr Build Mater. 2018 Sep;184(1):617-33, doi:10.1016/j.conbuildmat.2018.06.238.

7. Comité Euro-Internacional du Béton. CEB-FIP Model Code 1990. Structural Concrete: Textbook on Behaviour, Design and Performance. London: Thomas Telford; 1993.

8. Dias MM. Análise numérica de vigas mistas aço-concreto pelo método dos elementos finitos: efeitos de longa duração [dissertação]. Porto Alegre (RS): Universidade Federal do Rio Grande do Sul; 2013.177 p.

9. Bazant ZP, Prasannan S. Solidification theory for concrete creep. J Eng Mech. 1989 Aug;115(8):16911725.

10. Lazzari PM. Simulação numérica das etapas construtivas de pontes estaiadas através do método dos elementos finitos [tese]. Porto Alegre (RS): Universidade Federal do Rio Grande do Sul; 2016. 301 p. 
11. Quevedo FPdaM. Comportamento a longo prazo de túneis profundos revestidos com concreto: modelo em elementos finitos. Porto Alegre (RS): Universidade Federal do Rio Grande do Sul; 2017. 210 p.

12. Schmitz RJ. Estrutura mista aço-concreto: análise de ponte composta por vigas de alma cheia [dissertação]. Porto Alegre (RS): Universidade Federal do Rio Grande do Sul; 2017. 214 p.

13. Moreno JCA. Análise numérica de vigas mistas aço-concreto pelo método dos elementos finitos: modelos para os efeitos de longa duração e protensão interna [dissertação]. Porto Alegre (RS): Universidade Federal do Rio Grande do Sul; 2016.

14. Ross A. Creep of concrete under variable stress. J ACI. 1958 Mar;29(9):407-13.

15. Comité Euro-Internacional du Béton. CEB-FIB Model Code 2010. Lausanne; 2010.

16. Westerberg B. Time-dependent effects in the analysis and design of slender concrete compression members [thesis]. Stockholm (SWE): KTH, Trita-BKN. Bulletin, 94; 2008. 283 p.

17. ANSYS, Inc. Element Reference. Release 15.0; 2013. 